\title{
Unternehmensübergreifende Lernallianzen: Neue Pfade in der dualen Berufsausbildung
}

Die Versorgung der deutschen Wirtschaft mit qualifizierter Facharbeit wird auch künftig eine Grundlage für ökonomische Wettbewerbsfähigkeit unter globaler Konkurrenz bleiben. In der Metall- und Elektroindustrie mit ihrem Bedarf an hoch qualifizierten Fachkräften gibt es gute Erfahrungen mit unternehmensübergreifenden Lernallianzen, die auf eine langfristige Personalentwicklung durch berufliche Erstausbildung im Verbund abzielen. Kann das duale Berufsausbildungssystem so dem weitreichenden, beschleunigten Wandel in der Arbeitswelt begegnen? Wodurch zeichnen sich unternehmensübergreifende Lernallianzen im Hinblick auf künftige Qualifikationsanforderungen in modernen Produktionsprozessen aus?

KLAUS SCHMIERL

1. Korrespondenz zwischen Produktionsprozessen und dem Berufsausbildungssystem

Im vorliegenden Beitrag werden zwei Blickwinkel eingenommen. In einer Sicht auf die duale Berufsausbildung wird die Frage gestellt, wie das deutsche Berufsbildungssystem dem weitreichenden Wandel in der Industrie begegnen kann oder wird und welche Rolle dabei unternehmensübergreifende Lernallianzen spielen. In einer zweiten, arbeitspolitischen Perspektive wird die Frage behandelt, welche Bedeutung unternehmensübergreifende Lernallianzen also Ausbildungskooperationen oder Ausbildungsverbünde von mehreren rechtlich selbstständigen Unternehmen - für die Herausbildung von beruflichen Qualifikationen zur Bewältigung künftiger Anforderungen in der gewandelten Arbeitswelt haben.

\subsection{Wandel im Produktionsmodell, Fachkräfte- mangel und duale Berufsausbildung}

Das deutsche Innovations- und Produktionsmodell basiert auf dem Einsatz von qualifizierter Facharbeit. Das nach wie vor vorherrschende Modell zur Rekrutierung von Fachkräften ist die berufliche Erstausbildung und Weiterbildung innerhalb des dualen Systems (Cortina et al. 2003; Bosch 2010;
BIBB 2011a; BMBF 2011). Berufsausbildung ist insofern Resonanzboden der Produktionsweise und soll sicherstellen, dass den Unternehmen in Abhängigkeit vom nationalen Produktions- und Innovationsmodell in ausreichendem Maße sowie in passfähiger Qualität und Ausprägung Arbeitsund Fachkräfte zur Verfügung stehen. Dies ist jedoch umso schwieriger, je turbulenter das wirtschaftliche Umfeld ist bzw. je kurzfristiger und weitreichender sich die Arbeitswelt und der Produktionsmodus wandeln (Moldaschl et al. 2007).

In der soziologischen Arbeits- und Industrieforschung stellen wir gegenwärtig (und schon seit Längerem) eine Reihe von Entwicklungen fest: Die Richtung des Wandels von Arbeits- und Produktionsprozessen, die künftigen Formen von Arbeitseinsatz und Arbeitsorganisation sowie die hierfür benötigten Qualifikationen, Kompetenzen und Leistungsdimensionen der Arbeitskräfte lassen sich nicht mehr eindeutig prognostizieren. Diese Ungewissheit wird mit der Formulierung einer zunehmenden „Heterogenität von Produktionsprozessen“ auf den Begriff gebracht. Die Prozesse des Wandels finden in Gestalt immer kurzfristigerer Phasen betrieblicher Restrukturierungen und Neuzuschnitte von Unternehmen sowie eines erhöhten Innovationsdrucks statt. Die Marktanforderungen verändern sich rasant, die Unternehmen sind verstärkt in unübersichtliche globale Wertschöpfungsketten eingebunden und weitreichende Entscheidungen über die Zukunft eines Betriebes oder einer Arbeitsstätte werden gar nicht mehr vor Ort getroffen. Die steigende Dynamik und die damit verbundene Ungewiss- 
heit zukünftiger Anforderungen an Zahl und Qualifikation von Mitarbeitern machen es den Unternehmen schwer, Qualifikationsbedarfe zu ermitteln und systematische Ansätze zur Bewältigung der veränderten Anforderungen zu entwickeln.

Schließlich wurden in den vergangenen Jahren seitens der Politik den Arbeitgebern im Hinblick auf Arbeitsvertrags- und Beschäftigungsformen vielfältige Möglichkeiten eingeräumt, sich durch den Abschluss befristeter Arbeitsverträge oder auch durch die verstärkte Nutzung von Leiharbeit externe Flexibilisierungspotenziale zu verschaffen. Diese Entwicklung stellt die Stammbelegschaften selbst vor erhöhte Flexibilitätsanforderungen bzw. verschärfte Beschäftigungsrisiken.

Diese Veränderungen zeitigen weitreichende Konsequenzen für die Unternehmen hinsichtlich der Möglichkeiten und Verfahrensweisen zur Versorgung mit qualifizierter Arbeitskraft. Angesichts des zeitlich beschleunigten und räumlich entgrenzten Wandels mehren sich die Anzeichen, dass die Passfähigkeit des Berufsausbildungssystems in manchen Wirtschaftszweigen an seine Grenzen gelangen wird. In diesem Zusammenhang wird in quantitativen Studien für viele deutsche Industriebranchen in nächster Zukunft ein drastischer Fachkräftemangel prognostiziert. So stellte kürzlich das Institut der deutschen Wirtschaft Köln (IW) - aber auch der Deutsche Industrieund Handelskammertag (DIHK) und Prognos - fest, dass gegenwärtig vor allem in den MINT-Berufen (Mathematik, Informatik, Naturwissenschaften, Technik) in vielen Branchen eine große Lücke besteht (Anger et al. 2012). Und die Bundesagentur für Arbeit (2011, S. 6) erkennt zwar derzeit keinen generellen Fachkräftemangel, bestätigt aber schon heute nachweisliche Engpässe an Fachkräften in einzelnen Regionen Deutschlands und in bestimmten Berufen. Wenngleich damit ein durchgängiger Fachkräftemangel eine teilweise umstrittene Diagnose darstellt, richten sich Betriebe in ihrer Antizipation und ihren personalpolitischen Maßnahmen darauf ein. Zudem halten viele von uns befragten Unternehmen fest, dass die Anzahl von Schulabgängern und Bewerbern für ausgeschriebene Ausbildungsstellen in den letzten Jahren dramatisch abgenommen hat.

\subsection{Kooperation zwischen Unternehmen zur gemeinsamen Berufsausbildung}

Im deutschen System der dualen Berufsausbildung dauert es etwa drei bis vier Jahre nach erreichtem Schulabschluss, bis eine neue Generation von Ausgebildeten für die Wirtschaft bereitsteht. Wegen dieses zeitlichen Vorlaufs sind die Entscheider über Berufsausbildungsgänge und Curricula (wozu auch die Gewerkschaften zählen) vor das Erfordernis gestellt, künftige Veränderungen im Produktions- und Innovationsmodell zu antizipieren und im Hinblick auf den damit verknüpften Qualifikationsbedarf einzuschätzen. Angesichts der Reichweite der Veränderungen werden das
Konzept Beruflichkeit sowie die gemeinhin unterstellten Leitbilder von Berufsausbildung in ihrer bisherigen Form infrage gestellt: Betriebliche Restrukturierungen und Umsetzungen führen zu grundlegend anderen Anforderungen an die Qualifikation und die Flexibilität (im Arbeitsablauf sowie im Arbeitsort) der Mitarbeiter (vgl. Baethge et al. 2006; Bellmann et al. 2006). Sowohl für die Gesellschaft als Ganzes als auch für Unternehmen und Beschäftigte stellt sich hierbei eine Reihe von Anforderungen an rasche Investitionen in berufliche Qualifikation. Für die Unternehmen reicht es aufgrund der Veränderungen in den Arbeitsmarktbedingungen nicht mehr aus, sich am externen Arbeitsmarkt mit - zusehends knapper werdenden - ausgebildeten Fachkräften zu versorgen und auf deren adäquate Qualifikation und permanente Lernbereitschaft zu vertrauen. Für die Beschäftigten wiederum reicht es nicht aus, sich nach einem einmal erreichten qualifizierten Berufsabschluss auf die Flexibilitätspotenziale des eigenen Berufsbildes und eine lebenslange Beschäftigung beim selben Arbeitgeber im selben Berufsbild zu verlassen. Vielmehr verlangen die gegenwärtigen beschleunigten Veränderungsprozesse in der Arbeitswelt von beiden betrieblichen Akteuren, sich bei der Versorgung mit berufsfachlich ausgebildeten Arbeitskräften und in der Karriereentwicklung neu zu orientieren.

Gerade mit Blick auf die oben benannten Veränderungen wird dem deutschen Beschäftigungssystem und dualen Berufsausbildungssystem auch weiterhin eine hohe Flexibilität und eine große Reaktionsfähigkeit auf die Anforderungen des Wandels zugebilligt (Bosch 2010). Eine der Stärken des bundesdeutschen Beschäftigungsmodells liegt - darauf hat die nationale, aber auch die internationale Forschung hingewiesen - in der besonderen Ausprägung des beruflichen Erstausbildungssystems mit seiner Mischung aus theoretischer und praktischer Wissensvermittlung im Rahmen der dualen Berufsausbildung (vgl. Brater 2010; Hall/Soskice 2001). Gegenwärtig gibt es in Deutschland knapp 350 anerkannte Ausbildungsberufe (in allen Wirtschaftsbereichen), in denen sich betrieblich verwertbare Qualifikationen bündeln. Beruflich breit qualifizierte Beschäftigte sind funktional flexibel einsetzbar. Sie sind in der Lage, sich innerhalb des eigenen Berufsbildes Veränderungen in der Arbeitswelt anzupassen und damit zur Innovationsfähigkeit der Unternehmen beizutragen. Zudem erlaubt die breite Berufsausbildung auf dem externen Arbeitsmarkt eine "gesicherte Mobilität“; überwiegend münden die Erwerbsbiografien der beruflich Ausgebildeten in geschlossene Beschäftigungssysteme mit längerer Betriebszugehörigkeit und höherer Beschäftigungssicherheit (Struck/Dütsch 2012).

Dieser Beitrag geht der Frage nach, wie Unternehmen den Flexibilitätserfordernissen und drohenden Engpässen durch den Zuschnitt ihrer beruflichen Erstausbildung begegnen, um die für die Aufrechterhaltung der Produktionsund Arbeitsprozesse erforderlichen Qualifikationen in der benötigten Anzahl und im angemessenen qualitativen 
Zuschnitt zu beschaffen. Eine Antwort darauf sind unternehmensübergreifende Lernallianzen: In der Vergangenheit von uns durchgeführte Studien zu anderen Forschungsfragestellungen (vgl. Schmierl/Köhler 2007; Schmierl 2008) deuteten im Zusammenhang mit Anpassungen der betrieblichen Personalpolitik (überraschend) darauf hin, dass Unternehmen aus unterschiedlichen Branchen zunehmend allerdings bislang weitgehend von der Wirtschafts- und Sozialforschung vernachlässigt - Ausbildungskooperationen mit weiteren Akteuren und Firmen aus demselben Sektor oder derselben Region eingehen. Deshalb sind wir der Frage nach der Reichweite und Struktur dieser Lernallianzen in einem eigenen Forschungsvorhaben nachgegangen (Abschnitt 2.1).

\section{Erstausbildung in unternehmens- übergreifenden Lernallianzen}

Wie kann das Berufsbildungssystem adäquat mit dem weitreichenden, beschleunigten Wandel der Arbeitswelt korrespondieren? Ein wichtiger, in diesem Beitrag hervorgehobener Ansatzpunkt besteht darin, dass sich die Ausbildungsformen und -gänge der benötigten Offenheit anzunähern versuchen, indem sie geplant und systematisch den Umgang mit Flexibilität lehren und die Kooperationsfähigkeit sowie Prozessorientierung in den Mittelpunkt stellen. In der Metall- und Elektroindustrie ist dies seit der vor einigen Jahren vollzogenen Neuordnung der industriellen Metall- und Elektroberufe bereits bei der herkömmlichen Erstausbildung in den Betrieben dadurch gegeben, dass die Schulung zur Prozesskompetenz in der Berufsausbildung eine massive Aufwertung erfahren hat. Prozesskompetenz - und Teamfähigkeit - der Auszubildenden wird, wie unsere empirischen Untersuchungen zeigen, in unternehmensübergreifenden Lernallianzen besonders gefördert. Dies hängt mit der kollaborativen und flexiblen Struktur von Ausbildungskooperationen zusammen.

\subsection{Fokus der Forschung: Strategien zur Fachkräfteversorgung}

Während in statistischen Studien für die letzten Jahre eine gravierende Zunahme sogenannter prekärer Beschäftigungsverhältnisse festgestellt wird, verfolgen viele Unternehmen bewusst eine alternative Strategie: Sie versuchen, den Kernbestand an Fachkräften im Unternehmen und deren langfristige Rekrutierung durch eine angepasste Personal- und Organisationsentwicklung sowie eine Neuausrichtung der Versorgung mit qualifizierter Facharbeit sicherzustellen. Dem Herausarbeiten dieser Strategien und dem Identifizieren von innovativen Modellen einer modernen betrieblichen Personalentwicklung in Unternehmen sowie von deren Voraussetzungen widmete sich in den letzten drei Jahren das vom Bundesministerium für Bildung und Forschung (BMBF) geförderte Verbundprojekt „Beruflichkeit, Organisations- und Personalentwicklung im Spannungsfeld von Restrukturierung und Kompetenzsicherung - BOPS“ (Voss-Dahm et al. 2011). ${ }^{\bullet}$ Wir haben uns im Rahmen dieser Forschungsfrage auf die Metall- und Elektroindustrie konzentriert, wo die zentralen empirischen Feldphasen in den Jahren 2010 und 2011 stattfanden.

In unserem Teilprojekt haben wir Strukturen, Konstruktionsprinzipien und personalpolitische Kennzeichen von unternehmensübergreifenden Lernallianzen untersucht. Der Untersuchungsgegenstand waren eigeninitiativ gegründete Ausbildungsverbünde in der deutschen Metall- und Elektroindustrie, die ohne staatliche oder verbandliche Förderung entstanden sind. Unternehmensübergreifende Lernallianzen sind Ausbildungskooperationen oder Ausbildungsverbünde von mehreren rechtlich selbstständigen Unternehmen. Sie stellen besonders in der von Facharbeit und Facharbeitern geprägten Metall- und Elektroindustrie ein innovatives Modell dar, um eine Versorgung der Unternehmen mit Fachkräften langfristig zu sichern. Dies gilt speziell für kleine und mittelständische Unternehmen. Ihnen ist es oft nicht möglich, eigenständig und dauerhaft eine komplette und umfassende Erstausbildung bereitzustellen, weil es ihnen - auch aufgrund mangelnder finanzieller Ressourcen - an der notwendigen betrieblichen, organisatorischen, personellen oder technischen Infrastruktur für eine eigene Erstausbildung fehlt. So treten diese Unternehmen in Ausbildungsnetzwerke mit anderen Unternehmen ein, um gemeinsam Auszubildende zu schulen, die nach erfolgter Ausbildung prinzipiell in den Unternehmen übernommen werden. Es wurden zwei Intensivfallstudien und fünf Betriebsfallstudien in großen Ausbildungsverbünden mit langjähriger Kooperationserfahrung durchgeführt. Wir haben dabei in den Ausbildungsbetrieben und in den Stammbetrieben der Auszubildenden mit Personalleitern, Ausbildungsleitern, Ausbildungsbeauftragten, Ausbildern, Betriebsräten und Auszubildenden gesprochen und Betriebsbegehungen durchgeführt. Hinzu kamen Expertengespräche mit Vertretern von Wirtschaftsverbänden, Kammern und wissenschaftlichen Instituten und die Sichtung der einschlägigen Literatur (Schmierl 2011).

Das BOPS-Projekt wird seit Juni 2009 aus Mitteln des BMBF im Rahmen der Förderinitiative „Balance von Flexibilität und Stabilität in einer sich wandelnden Arbeitswelt" gefördert (Teilvorhaben des ISF München: „Unternehmensübergreifende Lernallianzen“, Förderkennzeichen 01FH09017). Betreut wird das Projekt vom Projektträger im DLR Arbeitsgestaltung und Dienstleistungen. Das Verbundprojekt wird koordiniert vom Institut Arbeit und Qualifikation (IAQ) an der Universität Duisburg-Essen; beteiligt sind als geförderte Partner zudem die Universität Bamberg und das Universitätsklinikum Köln. Die Verantwortung für den Inhalt dieses Beitrags liegt alleine beim Autor. 


\subsection{Personalpolitikvariante Lernallianz}

An der Personalpolitikvariante „Unternehmensübergreifende Lernallianzen" lässt sich eine Antwort auf die Frage untersuchen, wie die Herstellung qualifizierter Facharbeit weiterhin im Betrieb stattfinden kann. Zudem kann hier gezeigt werden, welche organisatorischen Voraussetzungen innerhalb des Betriebs und in überbetrieblichen Kooperationen heutzutage notwendig sind, um den Aufbau, den Erhalt und die Weiterentwicklung von beruflicher Qualifikation zu ermöglichen und abzusichern. Diese Kooperationsbereitschaft geht oftmals auf Schwierigkeiten bei der Rekrutierung von Facharbeitern zurück, etwa wenn spezifische Qualifikationen nicht in ausreichender Zahl und Qualität von den staatlichen Institutionen der beruflichen Ausbildung und Weiterbildung hervorgebracht werden. Vielfach hängt sie auch mit besonderen Schwierigkeiten eines bestimmten Typus von Unternehmen (wie kleine und mittlere (KMU) oder Unternehmen in Regionen mit besonderen Versorgungsengpässen) zusammen: Den jungen Arbeitsplatzsuchenden sind die Berufsprofile in diesen Unternehmen relativ unbekannt und sie entwickeln daher keine entsprechende Ausbildungsorientierung.

Die Unternehmen reagieren auf diese Rekrutierungsschwierigkeiten mit unterschiedlichen Initiativen. Viele intensivieren in herkömmlicher Weise ihre praktischen Weiterbildungsaktivitäten, um neues (z. T. ursprünglich nicht fachlich ausgebildetes) Personal besser zu integrieren und zu nutzen. Andere Unternehmen bauen, oftmals in Zusammenarbeit mit Arbeitsagenturen, eigene Lehrlingssysteme auf, in denen die Absolventen öffentlich geprüft und zertifiziert werden. Dieser Typus scheint im Ausland, wo kein duales Ausbildungssystem wie in der Bundesrepublik Deutschland vorhanden ist (wie in Schweden, Spanien und Irland), allgemein verbreitet zu sein. Wiederum andere Firmen verfolgen eine innovative Strategie der institutionalisierten Kooperation im Rahmen gemeinsamer Ausbildungsnetzwerke mit anderen Unternehmen innerhalb der Region und mit vergleichbaren Ausbildungsabschlüssen und Berufsprofilen. Letztere stehen in dieser Erörterung im Mittelpunkt.

Vor allem zwei Typen von Lernallianzen haben wir unter den erfolgreich praktizierten Ausbildungsverbünden der Metall- und Elektroindustrie gefunden. Es überwiegt bei Weitem der Typus „Auftragsausbildung“, gefolgt vom Modell „Leitbetrieb mit Partnerbetrieben“. Zusammen machen sie weit mehr als drei Viertel aller bestehenden Lernallianzen aus (siehe ausführlich Schmierl 2011). Bei der Auftragsausbildung findet eine Kooperation von mehreren Stammbetrieben mit einem Ausbildungsbetrieb statt. Der Stammbetrieb ist als „Arbeitgeber" Hauptansprechpartner für die Bewerber um Ausbildungsstellen im Vorfeld - bezüglich Vertragsabsprachen, Auswahl, Ausbildungsvertrag und Ausbildungsvergütungen. Die meisten Ausbildungsphasen finden aber im „fremden“ Ausbildungsbetrieb statt, der über eine Lehrwerkstatt verfügt. Die Gesamtverantwor- tung für den Auszubildenden (Azubi) hat der Stammbetrieb, die Verantwortung für die Ausbildung hat der Ausbildungsbetrieb. In der Praxis reichen die Angebote des Ausbildungsbetriebs von einzelnen Modulen (Modulausbildung) bis hin zur Übernahme der gesamten ersten beiden Lehrjahre inkl. Prüfungsvorbereitungen (Kernausbildung). Die Kostenerstattung wird entweder über bilaterale Absprachen oder einen vorab geschlossenen Ausbildungsauftrag geregelt.

Beim Typus Leitbetrieb mit Partnerbetrieben ist der Leitbetrieb die autonome Entscheidungsinstanz im Netzwerk. Er ist zuständig für Auswahl der Auszubildenden, Abschluss des Ausbildungsvertrags, Ausbildungsort, Vorbereitung der Curricula, Koordination der Ausbildung sowie Außenvertretung gegenüber externen Institutionen. Die wesentlichen Ausbildungsinhalte der - überwiegend branchenbezogenen - Ausbildungsberufe werden im Leitbetrieb vermittelt. Vor allem in den späteren Phasen der Ausbildung vergibt der Leitbetrieb einzelne Ausbildungsmodule an die Partnerbetriebe und organisiert die Entsendung der Auszubildenden dorthin. In der Praxis bildet der Leitbetrieb oftmals über seinen Bedarf aus. Die Auszubildenden werden nach der Ausbildung in der Regel nach vorher vereinbarten Regeln an die Partnerbetriebe verteilt oder bewerben sich dort eigenständig. In beiden Typen haben sich diese unternehmensübergreifenden Lernallianzen in den letzten Jahren in eine innovative, aber auch voraussetzungsvolle Richtung weiterentwickelt: Die Lernprozesse werden insbesondere in den letzten Lehrjahren in praktische Arbeitsund Kooperationszusammenhänge in den Betrieben eingebettet.

\subsection{Kollektivgut, Konkurrenz und Stabilität}

Unternehmensübergreifende Lernallianzen sind in den letzten Jahren in vielfältigen Branchen und Regionen sowie in unterschiedlichsten institutionellen Formen entstanden. Derartige Ausbildungsnetzwerke entstehen aus dem $\mathrm{Zu}$ sammentreffen von Angebot an und Nachfrage nach Ausbildungskapazitäten in einer Region. Letztere erfolgt durch Unternehmen, die selbst nicht in vollem Umfang ausbilden können oder wollen, die jedoch ein hohes Interesse an einer langfristigen Versorgung mit qualifizierter Facharbeit haben und dafür zu zahlen bereit sind. Die Nachfrage wird durch einen ausbildungsfähigen Betrieb bedient, der Überschüsse an Ausbildungskapazitäten und einen qualifizierten Stamm an Ausbildern verzeichnet. Solche Lernallianzen mit Firmen aus demselben Sektor oder derselben Region werden von den beteiligten Unternehmen als Erfolg versprechend und zukunftsweisend beurteilt.

In einer durch Konkurrenz geprägten Arbeitsmarktsituation entstehen damit aber gleichzeitig Herausforderungen insbesondere für die beteiligten kleinen und mittleren Unternehmen (KMU), da sie sich als Unternehmen öffnen und - trotz weiter bestehender Konkurrenzbeziehungen mit den größeren „Netzwerkpartnern“ - neue Formen 
der Kooperation eingehen und Vertrauen entwickeln müssen; ein Sachverhalt, der in der Arbeitsmarktforschung als Freerider- und Poaching-Problem beschrieben wird. Wir haben uns in den empirischen Untersuchungen deshalb auch Fragen zum Umgang mit derartigen Konkurrenzmechanismen um knappe Fachkräfte und Bewerber gewidmet und herausgearbeitet, wie derartige Kooperationen in der Ausbildung trotzdem funktionieren. Es wird eben notwendig, die Personalentwicklungsstrategien und internen Strukturen anzupassen sowie die Netzwerkkooperation zu verbessern. Diese Verbünde sind mithin nicht frei von Widersprüchen und erfordern eine neue Balance zwischen Flexibilität und Stabilität. Darüber hinaus sind eine kurzfristige Orientierung und die langfristige Perspektive in Einklang zu bringen. Antworten auf die Frage nach der Konkurrenzeindämmung liegen einerseits in einer klaren auch vertraglich abgesicherten Verbundstruktur und einem expliziten Abwerbeverbot sowie andererseits in einem transparenten Entscheidungs- und Koordinationsverfahren im Ausbildungsverbund. Dem Risiko, dass ein Kollektivgut wie Fachkräfte von einer Gruppe ausgebildet und dann ggf. von einem einzelnen Partner im Verbund „weggeschnappt “ wird, wird in solchen Verbünden auch durch Sanktionsmechanismen (wie dem erklärten Verzicht auf künftige Zusammenarbeit), aber auch durch die freiwillige Bereitschaft der Verbundteilnehmer begegnet, den langfristigen Bestand des Verbunds nicht durch Interessenegoismus zu gefährden.

Die Stabilität von unternehmensübergreifenden Lernallianzen wird in den empirisch untersuchten Fällen durch einen kleinen, langfristig partizipierenden Kreis von meist größeren Mittelständlern gewährleistet, die jährlich eine kritische Masse an Azubis zur Grundlagenausbildung anmelden. Weitere Fixkostenreduzierungen ergeben sich durch punktuelle Ausweitungen des Verbunds und fallweise Teilnahme oder Ausbildungsmodulbuchungen von weiteren Betrieben in der Region. Im Fall eines im Projekt intensiv untersuchten Ausbildungsverbunds von Unternehmen aus der Automobil- und Zulieferindustrie erfolgt über die klassische Grundlagenausbildung hinaus eine intensive netzwerkartige Zusammenarbeit zwischen vier am Verbund beteiligten Kernunternehmen. Diese Kooperation wird über regelmäßig tagende Gremien, wechselseitigen Informationsaustausch zur Abstimmung über künftige Ausbildungsinhalte und zur Weiterentwicklung des Ausbildungsverbunds sowie über eine Rotation der Azubis zwischen den Kooperationsbetrieben im dritten Lehrjahr sichergestellt. Um diesen inneren Zirkel gruppiert sich in einem flexiblen Kooperationsmuster eine Vielzahl von nicht ständig partizipierenden Unternehmen, die in unregelmäßigen Abständen die Grundlagenausbildung oder eine Modulausbildung in Anspruch nehmen. Bei der Modulausbildung absolvieren die Azubis den Hauptteil ihrer Ausbildung im Stammunternehmen, während der Ausbildungsbetrieb lediglich einzelne, klar definierte Module wie Lehrgänge oder Prüfungsvorbereitung übernimmt. Insbesondere für kleine und mittlere Unternehmen ist die Auftragsausbildung eine einfach zu implementierende Kooperationsform, um duale Berufsausbildung zu gewährleisten und ihren künftigen Fachkräftebedarf zu decken. Aber auch für die Auszubildenden bietet dieser Typus von Lernallianzen Vorteile, da sie eine qualitativ hochwertige Erstausbildung und zugleich Einblick in die betriebliche Arbeitssituation und Unternehmenskultur der anderen Azubis erhalten.

\section{Personalpolitische Wirkungen und die Position der Betriebsräte zu Lernallianzen}

Wenn wir auf die letzten beiden Dekaden eigener Forschungsarbeit zurückblicken, haben wir nur sehr wenige Forschungsthemen bearbeitet, bei denen eine derartig weitreichende Interessenübereinstimmung zwischen Vertretern der Management- und Arbeitskräfteseite gegeben war wie im Fall der unternehmensübergreifenden Lernallianzen. Es werden hierbei offenbar von beiden Betriebsparteien personalstrategische Vorteile gesehen sowie eine positive $\mathrm{Zu}$ kunft für junge Berufseinsteiger erwartet.

\subsection{Vorwegnahme der künftig benötigten und abgeforderten Qualifikationen}

Die Deckung des Personalbedarfs befriedigt zugleich das Interesse an mittelfristiger Kapitalverwertung. Auch die Arbeitsmarktkonkurrenz zwischen Unternehmen wird mit diesen Ausbildungsverbünden eingehegt: Dem Ausbildungsbetrieb verschafft die im Verbund organisierte, quantitativ umfangreiche Erstausbildung eine langfristig planbare Auslastung seiner Ausbildungskapazitäten und eine Absicherung seines Ausbildungspersonals. In einem Fall konnten sogar die von den Partnerunternehmen bezahlten Ausbildungskosten in Neuanschaffungen von Maschinen für die Lehrwerkstätte reinvestiert werden. Die Stammbetriebe, die ihre jungen Azubis in den Ausbildungsbetrieb entsenden, haben interne Vorteile. Durch den Verzicht auf größere Investitionen und auf das Vorhalten von Ausbildungskapazitäten werden sie von Kosten entlastet. Zugleich profitieren sie auch von externen Vorteilen: Sie können die qualitativ spezialisierte und permanent geschulte Ausbildermannschaft im Ausbildungsbetrieb nutzen.

Wirft man einen sozialwissenschaftlichen Blick auf die Folgen für die sogenannten Matching-Prozesse, also die zu erwartende künftige Deckungsgleichheit zwischen den mitgebrachten Qualifikationen der Arbeitskräfte und den abgefragten Arbeitsanforderungen sowie benötigten Kompetenzen im Produktionsprozess, so besteht bei diesen unternehmensübergreifenden Lernallianzen eine besonders ausgeprägte Übereinstimmung. Der entscheidende Vorteil 
in Bezug auf die Vermittlung beruflicher Qualifikationen liegt in der hochgradigen Übereinstimmung der Lehrinhalte, der Lehranforderungen und der Kooperationserfordernisse mit den zukünftigen Aufgaben von qualifizierten Beschäftigten in modernisierten Arbeits- und Produktionsprozessen. Ausbildung in unternehmensübergreifenden Lernallianzen vermittelt damit in besonderer Weise die für die künftigen Anforderungen in Arbeits- und Produktionsprozessen benötigten Qualifikationen und nimmt diese gewissermaßen im Ausbildungsverfahren vorweg - in inhaltlicher, organisatorischer und methodischer Hinsicht:

- inhaltlich durch die Passung von zeitgemäßen, ganzheitlichen Ausbildungsinhalten und künftigen Arbeitsanforderungen, wie autonome Problemlösung und Selbstständigkeit (z. B. durch eigene betriebliche Produktionsaufträge); - organisatorisch durch Arbeit in einem größeren, evtl. interdisziplinären und sogar unternehmensübergreifenden Teamzusammenhang. Durch heterogene Herkunft der Azubis sowie betriebsübergreifende Erfahrungshintergründe und Koordinierungsanforderungen werden soziale Qualifikationen und koordinierende Fähigkeiten vorausgesetzt und zugleich intensiv gelehrt;

- methodisch durch das Erlernen von Teamfähigkeit und Projektmanagement, sozialer Koordinierung sowie Flexibilität und Selbstorganisation (z. B. durch Betriebsrotation).

Darüber hinaus erhalten die Azubis aufgrund von Professionalisierungseffekten eine Ausbildung auf hohem fachlichem Niveau. Diese wird gewährleistet durch eine moderne Ausstattung der Lehrwerkstätten sowie durch qualifizierte, hauptamtliche Ausbilder, die im Gegensatz zu ihren nebenamtlichen Pendants in einer klassischen Ausbildungsstätte sowohl über größere zeitliche Ressourcen verfügen als auch meist fachlich eher in der Lage sind, individuelle Schwächen der Azubis zu erkennen und gezielt auszumerzen. Einige Ausbildungsbetriebe fördern dies durch spezielle Weiterbildungen der Ausbilder in den Bereichen Kommunikation mit Jugendlichen, Teamentwicklung, Moderation, Konfliktmanagement etc. und bieten den Azubis als Ergänzung zur Berufsschule zusätzlichen Werkunterricht oder Nachhilfe an. Neben fachlichem Know-how wird in den untersuchten Fällen andererseits der gezielten Vermittlung von Methoden und persönlichen Kompetenzen ein hoher Stellenwert beigemessen. Mehrtägige Einführungsseminare abseits der Arbeitsumgebung (Outdoorseminare mit Rafting, Zelten, Schneeschuhtouren etc.) wie auch das gemeinsame Lernen der Azubis über mehrere Lehrjahre dienen dem Kennenlernen und der Stärkung der Teamfähigkeit. In einer sehr intensiv untersuchen Ausbildungspartnerschaft in der ostdeutschen Automobilindustrie bearbeiten die Azubis darüber hinaus eine ganze Woche lang ein regionales soziales Projekt (wie z. B. die Anfertigung einer Kletterburg für einen örtlichen Kindergarten oder eines Heuhotels für eine Jugendherberge), bei dem sie von der Projektplanung über die Materialbeschaffung und die Arbeitsplanung bis hin zur Übergabe an den Auftraggeber alles selbst organisieren und durchführen. Die Flexibilität der Azubis wird dadurch erhöht, dass sie sich - besonders wenn regelmäßig Betriebsrotationen zwischen Partnerunternehmen vorgesehen sind - auf unterschiedliche Führungsstile, Produktionstechniken und Unternehmenskulturen einstellen müssen. Die zunehmende Prozessorientierung in der Ausbildung festigt schließlich die Selbstständigkeit, Eigenverantwortung und Problemlösungskompetenz der Azubis, die frühzeitig umfassende Arbeitsprozesse von der Auftragsannahme bis zur Endkontrolle planen und eigenständig durchführen müssen. Das Ziel ist die bewusste und frühzeitige Vorbereitung der Azubis auf die im Arbeitsleben benötigten Kompetenzen.

Dies bestätigt sich auch in den empirischen Befragungen zur Interessenlage der Auszubildenden: Es fiel ihnen in den Interviews durchweg schwer, überhaupt Nachteile der unternehmensübergreifenden Lernallianz zu benennen. Dagegen sahen alle Azubis weitreichende individuelle Vorteile in der Verbundausbildung. Einige typische Aussagen:

- „Ein Vorteil an der Ausbildungspartnerschaft ist, dass man andere Azubis kennenlernt und neue Kontakte knüpfen kann.“

- „Man kann zwischenzeitlich auch durch die Fertigung des Ausbildungsbetriebs gehen. Und im Betriebsdurchlauf sieht man auch die Werke von den anderen drei Firmen.“ - „Hier in der Lehrwerkstätte sind mehrere Azubis, die im Team zusammenarbeiten müssen. Bei meinem eigenen Betrieb wären wir nur zwei Zerspaner im zweiten Lehrjahr und dann ja eigentlich kein Team.“

- „Hier kann man viel mehr und viel besser für die Prüfung lernen. Und in der Lehrwerkstatt kann ich bestimmte Dinge für die Prüfung nachholen. Im eigenen Unternehmen hätte ich fast gar nicht gefräst und gedreht, weil ich nicht aus einem Metallbetrieb komme.“

\subsection{Wertschätzung durch Personalleitungen und Betriebsräte}

$\mathrm{Zu}$ dieser Zufriedenheit der Auszubildenden kommt eine ausgeprägte Rückendeckung durch Personalleitungen und Betriebsräte. In den von uns untersuchten unternehmensübergreifenden Lernallianzen ergibt sich eine Win-winSituation zwischen dem Unternehmensmanagement und den betrieblichen Interessenvertretungen. Das gilt sowohl für die Ausbildungsbetriebe und Leitbetriebe als auch für die entsendenden Stammbetriebe.

Für die Personalleitungen bildet die Lernallianz nicht nur eine langfristig abzielende Strategie zur Sicherung des eigenen Fachkräftenachwuchses, sondern erlaubt auch die Etablierung eines Images als sozial engagierter Arbeitgeber in der Region. Hier lässt sich das hohe gesellschaftliche Prestige ablesen, das der Durchführung einer beruflichen Erstausbildung innewohnt. 
Die in allen Untersuchungsbetrieben vorhandenen und befragten Betriebsräte agieren sehr wohlwollend, stehen zumeist in engem, manchmal freundschaftlichem Kontakt zu den Ausbildern, Ausbildungsleitern und Ausbildungsbeauftragten und bilden gewissermaßen von Natur aus die strategischen und taktischen Koalitionspartner der Personalleitungen und Ausbildungsleitungen. Aus ihrer Sicht signalisiert das Unternehmen damit die hohe Wertschätzung einer qualifizierten und in der Regel langfristig beschäftigten Mannschaft, beweist aus gewerkschaftspolitischer Perspektive gesellschaftliche Verantwortung und setzt sich zugunsten einer Langfristperspektive in der Personalpolitik strategisch gegenüber kurzfristigen Profitmaximierungs- und Shareholder-Value-Orientierungen ab. Darüber hinaus erhalten die Betriebsräte aller Teilnehmerunternehmen durch eine solche Langfristinvestition der Betriebe in die Personalausbildung und -entwicklung gewissermaßen eine informelle Bestätigung, dass das eigene Unternehmen keine Standortverlagerungen vorbereitet. Dieser Befund mag angesichts dessen, welche Position zur Erstausbildung man sich von einem Betriebsrat und Gewerkschafter erwartet, weitgehend überraschungsfrei sein, verdeutlicht allerdings sehr gut den von unterschiedlichen Wirtschaftsparteien der dualen Berufsausbildung zugewiesenen gesellschaftlichen Stellenwert, wozu eben unternehmensübergreifende Lernallianzen einen zusätzlichen Baustein und Pfad anbieten.

Als ein weiterer interessenpolitisch wirksamer, aber auch für die Auszubildenden motivierender Effekt ließ sich in den von uns untersuchten Fällen eine wachsende Einbindung und Zusammenarbeit der verschiedenen betrieblichen Jugend- und Auszubildendenvertretungen (JAV) erkennen. So wurde mancherorts mit gemeinsamen Treffen und dem Aufbau von Kommunikationsstrukturen begonnen, um ggf. auch gemeinsam Veränderungen in den Ausbildungsinhalten oder der Organisation zu initiieren. Aus Betriebsratsperspektive kann es im Hinblick auf eine Angleichung von tariflichen und arbeitspolitischen Standards auch ein Vorteil sein, dass sich die Azubis über Ausbildungsbedingungen, Lehrlingsgehälter, Tarifbindungen und Einsatzbedingungen austauschen.

\section{Resümee}

In der Wissenschaft, seitens der Akteure der (Bildungs-) Politik, aber auch in den Personalabteilungen der Unternehmen wird immer deutlicher vorausgesehen, dass der deutschen Industrie in der allernächsten Zukunft ein - auch durch den demografischen Wandel verstärkter - Fachkräftemangel in massivem Ausmaß droht. Gerade in industriell geprägten Branchen mit hohem Innovationsgrad und starken Exportaktivitäten müssen sich die Unternehmen auf eine deutlich erschwerte Versorgung mit beruflich qualifi- zierten Fachkräften, aber auch ausbildungsbereiten Schulabsolventen einstellen (BMBF 2011, S. 25 und S. 34f.). Dies gilt in besonderem Maße für die Metall- und Elektroindustrie.

Das deutsche Beschäftigungs- und Berufsausbildungssystem mit seiner Orientierung auf flexibel und breit qualifizierte Fachkräfte sowie auf sozialpartnerschaftlich definierte praxisbezogene, zertifizierte Berufsbilder und seiner langfristig professionellen Ausrichtung weist zur Bewältigung dieser Anforderungen eine hohe Flexibilität und Reaktionsfähigkeit auf. Die berufliche Erstausbildung und Weiterbildung innerhalb des dualen Systems mit ihrer Kopplung von Theorie und Praxis ist nach wie vor das vorherrschende und auch gut funktionierende Modell zur Rekrutierung von Fachkräften. Für die betrieblichen Personalentwicklungsstrategien kommt nun mit den unternehmensübergreifenden Lernallianzen eine weitere Option zur Versorgung mit qualifizierter Facharbeit hinzu. In einer bewusst langfristig orientierten Ausbildungsperspektive wird qualifizierte Facharbeit mit solchen Modellen als das anerkannte „Rückgrat des deutschen Produktionsmodells“ und als Voraussetzung für eine primär auf Produktivität und Innovation zielende High-Road-Strategie gestützt (vgl. Voss-Dahm et al. 2011).

Schon die in der Berufsausbildung vor einigen Jahren vollzogene Neuordnung der industriellen Metall- und Elektroberufe stellte die Prozesskompetenz in den Mittelpunkt - eine wichtige Rahmenbedingung für die Zukunftsfähigkeit der Berufsausbildung. Deren Aneignung wird wiederum in unternehmensübergreifenden Lernallianzen intensiviert: Ist doch das Besondere daran, dass aufgrund der kooperativen Struktur und der teamspezifischen Lehrinhalte von Ausbildungspartnerschaften insbesondere die Prozesskompetenz eine ganz spezifische Prägung erfährt.

Gegenüber einer „klassischen“ Erstausbildung im Einzelbetrieb bieten Lernallianzen erweiterte Ausbildungschancen für die ausbildungswilligen Jugendlichen und die beteiligten Unternehmen, die in ihrer Eigenschaft als kooperierender Ausbildungsverbund begründet liegen. So können sich die Betriebe bei der Gewinnung von Auszubildenden wechselseitig unterstützen. Die Kontinuität der Ausbildung wird auch dann sichergestellt, wenn ein Verbundpartner zeitweise oder ganz ausfällt. Auszubildende erwerben fachliche und Schlüsselqualifikationen in besonderer Breite, Form und Qualität, die zugleich den Anforderungen der Partnerbetriebe besonders gut angepasst sind. Schließlich erhalten die Auszubildenden durch Hospitationen einen Einblick in die Strukturen und Führungsstile der anderen Partnerbetriebe und damit einen Vergleichsmaßstab für die Qualität der eigenen Ausbildungssituation und betrieblichen Betreuung. Unternehmen und Beschäftigte tragen somit zur Kontinuität des Prinzips Berufsfachlichkeit bei.

In der Debatte um die Zukunft und die Entwicklungsoptionen von qualifizierter Facharbeit in den Betrieben und auf dem Arbeitsmarkt lassen sich mit der Analyse moderner 
Personalentwicklungsstrategien wie den unternehmensübergreifenden Lernallianzen Antworten auf die Frage geben, wie sich das Prinzip „Berufsfachlichkeit" und das deutsche Berufsbildungssystem weiterentwickeln (können). D.h. auch, wie sich die duale berufliche Bildung im Spannungsfeld zwischen Flexibilitätszwängen und Stabilisierungserfordernissen auch in Zukunft behaupten kann. Die von uns empirisch analysierten Ansätze verweisen auf moderne Konzepte der beruflichen Bildung, die angesichts der Veränderungen in einer sich wandelnden Arbeitswelt zu einem sozial verträglichen Ausgleich im Betrieb beitragen sowie den Aufbau und langfristigen Erhalt von Qualifikationen sicherstellen können. Über diesen primär gesellschaftlichen Effekt hinaus bietet sich aber mit dem Erstausbildungsmodell „Unternehmensübergreifende Lernallianzen“ auch in arbeitspolitischer und beschäftigungspragmatischer Hinsicht eine Lösung (unter anderen) an, mit der der prognostizierte und in Teilen der hochqualifizierten Metall- und Elektroindustrie bereits eingetretene Fachkräftemangel künftig bewältigt werden kann. Bietet doch diese Erweiterung und Bereicherung des deutschen Systems der dualen Berufsausbildung gerade für die bislang ausbildungsabstinenten Unternehmen eine Möglichkeit, an einem Ausbildungsverbund zu partizipieren, indem sie die Erstausbildung (teilweise) delegieren und die künftige Fachkräftegeneration beim kooperierenden Ausbildungsbetrieb im Ausbildungsnetzwerk ausbilden lassen.

\section{LITERATUR}

Anger, Ch./Geis, W./Plünnecke, A. (2012): MINT-Frühjahrsreport 2012, Köln Baethge, M./Baethge-Kinsky, V./Holm, R./Tullius, K. (2006): Dynamische Zeiten - langsamer Wandel: Betriebliche Kompetenzentwicklung von Fachkräften in zentralen Tätigkeitsfeldern der deutschen Wirtschaft, Abschlussbericht, Göttingen

Bellmann, L./Bielenski, H./Bilger, F./Dahms, V./Fischer, G./Frei, M./Wahse, J. (2006): Personalbewegungen und Fachkräfterekrutierung. Ergebnisse des IABBetriebspanels 2005, IAB Forschungsbericht (11), Nürnberg

Bosch, G. (2010): Strukturen und Dynamik von Arbeitsmärkten, in: Böhle, F./ Voß, G./Wachtler, G. (Hrsg.): Handbuch Arbeitssoziologie, Wiesbaden,

S. $643-670$

Brater, M. (2010): Berufliche Bildung, in: Böhle, F./Voß, G./Wachtler, G. (Hrsg.): Handbuch Arbeitssoziologie, Wiesbaden, S. 805-837
Bundesagentur für Arbeit (BA) (2011): Perspektive 2025: Fachkräfte für Deutschland, Nürnberg

Bundesinstitut für Berufsbildung (BIBB) (2011a): Datenreport zum Berufsbildungsbericht 2011, Bonn

Bundesinstitut für Berufsbildung (BIBB) (2011b): Verbundausbildung - vier Modelle für die Zukunft, Jobstarter Praxis, Bd. 6, Bonn Bundesministerium für Bildung und Forschung (BMBF) (Hrsg.) (2011): Berufsbildungsbericht 2011, Bonn

Cortina, K./Baumert, J./Leschinsky, A./Mayer, K./Trommer, L. (Hrsg.) (2003):

Das Bildungswesen in der Bundesrepublik Deutschland, Reinbek

Hall, P./Soskice, D. (2001): Varieties of Capitalism: The Institutional Framewok of Comparative Advantage, Oxford

Moldaschl, M./Ludwig, J./Schmierl, K. (2007): Arbeitsforschung und Innovationsfähigkeit in Deutschland, in: J. Ludwig, M./Moldaschl, M./Schmauder, K./ Schmierl (Hrsg.): Arbeitsforschung und Innovationsfähigkeit in Deutschland, München/Mering, S. 11-19

Schmierl, K. (2008): Eine neue AERA in Thüringen - Einführung und Umsetzung des Entgeltrahmenabkommens in ausgewählten Pilotbetrieben, ISF Forschungsberichte, München

Schmierl, K. (2011): Unternehmensübergreifende Lernallianzen in der Metallund Elektroindustrie -Typologie, Besonderheiten und theoretische Implikationen, in: Voss-Dahm, D. et al., a.a.O., S. 25-48

Schmierl, K./Köhler, H.-D. (2007): Organisationslernen in Lowtech und Medium-Lowtech-Unternehmen: Wissens- und Personalmanagement, in: Abel, A./ Hirsch-Kreinsen, H. (Hrsg.): Lowtech-Unternehmen am Hightech-Standort, Berlin

Struck, O./Dütsch, M. (2012): Gesicherte Mobilität: Zur Bedeutung berufsfachlicher Qualifikationen in geschlossenen und offenen Beschäftigungssystemen, in: D. Alewell/O. Struck (Hrsg.): Betriebliche Beschäftigungssysteme, Sonderheft Industrielle Beziehungen, Mering, S. 154-186

Voss-Dahm, D./Mühge, G./Schmierl, K./Struck, O. (Hrsg.) (2011): Qualifizierte Facharbeit im Spannungsfeld von Flexibilität und Stabilität, Wiesbaden

\section{AUTOR}

KLAUS SCHMIERL, Dr., Wissenschaftler am Institut für Sozialwissenschaftliche Forschung e.V. (ISF München). Arbeitsschwerpunkte: Industrielle Beziehungen, Lohn- und Tarifpolitik, Berufe und Qualifikationsentwicklung, Internationalisierung von Unternehmen und interkulturelle Arbeit.

klaus.schmierl@isf-muenchen.de 